\title{
Direct detection and solar capture of spin-dependent dark matter
}

\author{
Zheng-Liang Liang* and Yue-Liang $\mathrm{Wu}$ \\ State Key Laboratory of Theoretical Physics (SKLTP), \\ Kavli Institute for Theoretical Physics China (KITPC), \\ Institute of Theoretical Physics, Chinese Academy of Science, Beijing 100190, China and \\ University of Chinese Academy of Sciences, Beijing 100049, China
}

\begin{abstract}
We investigate the implication of different elastic spin-dependent (SD) operators on both the direct and indirect detections of the weakly interacting massive particle (WIMP). Six representative building blocks of SD operators, together with their counterparts with a massless mediator, are considered to interpret the direct detection experiments (Xenon100, SIMPLE, and COUPP) in a comprehensive way. We also study the solar capture and annihilation of WIMPs with these effective SD operators and place the constraints on the relevant annihilation rate from neutrino detection experiments Super-Kamionkande and IceCube. Upper limits on the WIMP-nucleon couplings drawn from direct detections are also projected to the annihilation rate for contrast and complementarity. We find that the efficiency of these mentioned detection strategies depends specifically on the six SD operators, while the neutrino-based detections are more effective in exploring the parameter space for the massless mediator scenario.
\end{abstract}

${ }^{*}$ Electronic address: liangzl@itp.ac.cn

$\dagger$ Electronic address: ylwu@itp.ac.cn 
The existence of dark matter (DM) has been well confirmed through decades of endeavor in cosmological observation [1, 2]. However, the nature of the DM still remains a challenging problem for particle physics. Among those mechanisms and DM candidates, the weakly interacting massive particle (WIMP) is one of the most promising. In this picture, the WIMPs interact with the standard model particles through weak interactions and naturally result in the thermal relic density consistent with the observation, called the "WIMP miracle." Both theoretical and experimental attempts have been made to account for and search for such particles. Many direct detection groups have reported their results based on the conventional elastic spin-independent (SI) or spin-dependent (SD) effective operator that is proportional to a constant, $\mathcal{M}_{\mathrm{SI}} \propto 1$, or to the dot product between the spins of the WIMP and quark, $\mathcal{M}_{\mathrm{SD}} \propto \mathbf{S}_{\chi} \cdot \mathbf{S}_{q}$. However, if we broaden our study to more general DM scenarios such as isospin-violating DM [3], long-range force DM [4, 5], inelastic DM [6], and form factor DM [7], the interpretation of the experimental results may turn out to be quite different. This possibility has been used in attempts to alleviate the conflicts between different direct detection experiments [8 18].

In recent years, several groups began to systematically study the DM direct detection in terms of the nonrelativistic (NR) effective field theory [19 22], in which a given Lorentzinvariant interaction is expanded with a complete set of NR effective operators at the nucleon level, along with the corresponding Galilean and rotational invariant coefficients. The authors of Ref. [20] have further calculated the relevant nuclear form factors associated with different effective operators beyond the simplest SI and SD case, for the elements common in the present-day detectors. Besides, a state-of-the-art large-scale nuclear structure calculation for different operators has also been planned in [23]. Further knowledge about the nuclear form factors is important for us to interpret the detection results in a more comprehensive way.

Besides direct detection efforts, some indirect approaches are also expected to be effective in constraining the coupling strength between the WIMP and nucleon [24 27]. One may regard the solar neutrinos that are detected by the neutrino detectors (e.g., SuperKamionkande [28] or IceCube [29, 30]) as the possible annihilation products of the trapped WIMPs residing in the center region of the Sun, so as to impose upper limits on the WIMPnucleus coupling coefficients, which is relevant to the WIMP capture rate and thus to the neutrino flux, if an equilibrium between capture and annihilation is assumed. 
The implications of a wide variety of elastic SI interactions on both the direct and indirect detection experiments have been studied in Ref. [31], in which the direct detections are proved to be more effective in constraining the WIMP-nucleon interaction couplings with a sensitivity $2 \sim 4$ orders of magnitude greater than the neutrino-based approach. However, given that the upper limit on the elastic SD WIMP-nucleon cross section is far above that of the SI interaction, one would expect the neutrino-based detection to compete with or even exceed the direct detections in sensitivity for effective SD operators. We will study in this paper the direct detection of the WIMPs with a set of effective elastic SD operators, as well as the relevant indirect detection in a similar way with [31] and compare them with each other in sensitivity. We organize these discussions as follows: In Sec. I we discuss the effective SD operators in a systematic manner and the relevant consequences for various WIMP direct detection experiments. In Sec. II we study the solar capture of the WIMP in detail and calculate the bounds on the capture rate $C_{\odot}$, and the annihilation rate $\Gamma_{\odot}=C_{\odot} / 2$, imposed by both the direct detection experiments and the neutrino detectors Super-Kamionkande and IceCube, for the purpose of comparison and complementarity. Conclusions and discussions are given in Sec. III.

\section{DARK MATTER DIRECT DETECTION}

\section{A. Direct detection recoil rate}

In general the differential event rate $R$ in a direct detection experiment is given as an average over the WIMP distribution,

$$
\frac{d R}{d E_{R}}=N_{T} \frac{\rho_{\chi}}{m_{\chi}} \int_{v_{\min }}^{v_{\mathrm{e}}} \frac{d \sigma}{d E_{R}} v f(\mathbf{v}) d^{3} v,
$$

where $N_{T}$ is the effective number of target nucleus in the detector, and $\rho_{\chi}$ is the local WIMP halo density in our earth neighborhood, with $m_{\chi}$ being the WIMP mass. The WIMP velocity distribution $f(\mathbf{v})$ is defined in the laboratory reference frame with the incident WIMP velocity $\mathbf{v}$, and $d \sigma / d E_{R}$ is the relevant WIMP-nucleus differential cross section, which can be further expressed (by summing over initial spins and averaging over finial spins) as 


$$
\frac{d \sigma}{d E_{R}}=\frac{m_{T}}{2 \pi v^{2}} \frac{1}{(2 J+1)\left(2 s_{\chi}+1\right)} \sum_{\text {spins }}\left|\mathcal{M}_{\mathrm{NR}}\right|^{2} .
$$

We denote the nucleus spin and WIMP spin as $J$ and $s_{\chi}$, respectively, and $m_{T}$ is the mass of the target nucleus. $\mathcal{M}_{N R}$ is the nonrelativistic scattering amplitude, which differs from the relativistic one $\mathcal{M}$ by $\mathcal{M}_{N R}=\mathcal{M} /\left(4 m_{\chi} m_{T}\right)$. Thus by Eq. (2), Eq. (11) can be further written as

$$
\frac{d R}{d E_{R}}=N_{T} \frac{\rho_{\chi}}{m_{\chi}} \int_{v_{\min }}^{v_{\mathrm{e}}} \frac{m_{T}}{2 \pi} \frac{1}{(2 J+1)\left(2 s_{\chi}+1\right)} \sum_{\text {spins }}\left|\mathcal{M}_{\mathrm{NR}}\right|^{2} \frac{f(\mathbf{v})}{v} d^{3} v .
$$

The upper limit of integral $v_{e}$ is the galactic escape velocity relative to the detector, and the lower limit $v_{\min }$ is the minimal possible velocity for a fixed recoil energy $E_{R}$, which is related to the nucleus mass $m_{T}$ and reduced mass $\mu_{T}$ of the WIMP-nucleus pair through

$$
v_{\min }=\frac{q}{2 \mu_{T}}
$$

where $q=\sqrt{2 m_{T} E_{R}}$ is the transferred momentum for elastic scattering.

\section{B. Elastic spin-dependent dark matter form factors}

Instead of listing possible Lorentz-invariant effective operators in our model-independent analysis, we take an alternative strategy by following [32] to enumerate a set of effective operators at the nonrelativistic level, which preserve Galilean invariance and rotational symmetry degenerated from Lorentz symmetry, as well as their corresponding discrete symmetry at the relativistic level. These independent 15 operators with their invariant coefficients exhausting all possible nonrelativistic effective operators for spin 1/2 WIMP, which originate from the nonrelativistic reduction of all possible 20 bilinear amplitude products [20, 32], may also be encountered in higher-spin WIMP models. We first divide the following 11 effective WIMP-nucleon operators into the following six groups separately in six lines:

1. P-even, $\mathbf{S}_{\chi}$-independent, T-even

$$
\mathcal{O}_{1, N}=1, \quad \mathcal{O}_{2, N}=\left(v^{\perp}\right)^{2}, \quad \mathcal{O}_{3, N}=i \mathbf{S}_{N} \cdot\left(\mathbf{q} \times \mathbf{v}^{\perp}\right),
$$


2. P-even, $\mathbf{S}_{\chi}$-dependent, T-even

$$
\mathcal{O}_{4, N}=\mathbf{S}_{\chi} \cdot \mathbf{S}_{N}, \quad \mathcal{O}_{5, N}=i \mathbf{S}_{\chi} \cdot\left(\mathbf{q} \times \mathbf{v}^{\perp}\right), \quad \mathcal{O}_{6, N}=\left(\mathbf{S}_{\chi} \cdot \mathbf{q}\right)\left(\mathbf{S}_{N} \cdot \mathbf{q}\right)
$$

3. P-odd, $\mathbf{S}_{\chi}$-independent, T-even

$$
\mathcal{O}_{7, N}=\mathbf{S}_{N} \cdot \mathbf{v}^{\perp}
$$

4. P-odd, $\mathbf{S}_{\chi}$-dependent, T-even

$$
\mathcal{O}_{8, N}=\mathbf{S}_{\chi} \cdot \mathbf{v}^{\perp}, \quad \mathcal{O}_{9, N}=i \mathbf{S}_{\chi} \cdot\left(\mathbf{S}_{N} \times \mathbf{q}\right)
$$

5. P-odd, $\mathbf{S}_{\chi}$-independent, T-odd

$$
\mathcal{O}_{10, N}=i \mathbf{S}_{N} \cdot \mathbf{q}
$$

6. P-odd, $\mathbf{S}_{\chi}$-dependent, T-odd

$$
\mathcal{O}_{11, N}=i \mathbf{S}_{\chi} \cdot \mathbf{q}
$$

where $\mathbf{q}$ is the momentum transferred to the WIMP. It is easy to verify that each operator from one group does not interfere with operators from another by the consideration of symmetry and the WIMP spin $\mathbf{S}_{\chi}$. In addition we list another three operators that are constructed from the mixing among the above operators,

$$
\mathcal{O}_{10, N} \mathcal{O}_{8, N}, \mathcal{O}_{11, N} \mathcal{O}_{7, N}, \mathcal{O}_{11, N} \mathcal{O}_{3, N}
$$

and one last building block to form a complete set,

$$
\mathcal{O}_{12, N}=\mathbf{S}_{\chi} \cdot\left(\mathbf{S}_{N} \times \mathbf{v}^{\perp}\right)
$$

The Hermitian "perpendicular" operator $\mathbf{v}^{\perp}=\mathbf{v}+\frac{\mathbf{q}}{2 \mu_{N}}$ satisfies $\mathbf{v}^{\perp} \cdot \mathbf{q}=0$ when the on-shell condition is imposed, and $\mathbf{v}$ is the velocity of the WIMP with respect to the nucleon. One should note that only SD operators $\mathcal{O}_{3, N}, \mathcal{O}_{4, N}, \mathcal{O}_{6, N}, \mathcal{O}_{7, N}, \mathcal{O}_{9, N}$, and $\mathcal{O}_{10, N}$ are included in 
our study in this work, because these element operators are not only frequently encountered in practical operator expansion but also sufficient to give us a general description on how a variety of SD effective operators with different powers of $q$ and $v^{\perp}$ will lead to different interpretations of DM detection experiments. By the notation of [20], the differential recoil rate for the conventional $\mathrm{SD}$ operator $\mathcal{O}=a_{p} \mathbf{S}_{\chi} \cdot \mathbf{S}_{p}$ is written as

$$
\frac{d R}{d q}=N_{T} \frac{\rho_{\chi}}{m_{\chi}} \frac{16}{3} \frac{\sigma_{p}}{2 \mu_{p}^{2}} \frac{\left(F_{\Sigma^{\prime \prime}}^{(p, p)}+F_{\Sigma^{\prime}}^{(p, p)}\right)}{16} q \int_{v_{\min }}^{v_{\mathrm{e}}} \frac{f(\mathbf{v})}{v} d^{3} v
$$

and the WIMP-proton cross section $\sigma_{p}$ and coupling $a_{p}$ are connected to each other by

$$
\frac{\sigma_{p}}{2 \mu_{p}^{2}}=C\left(s_{\chi}\right) \frac{a_{p}^{2}}{2 \pi} \frac{3}{16}
$$

where $F_{\Sigma^{\prime}}^{(p, p)}$ and $F_{\Sigma^{\prime \prime}}^{(p, p)}$ are the transverse and the longitudinal form factors respectively [20], and $C\left(s_{\chi}\right)=\frac{4}{3} s_{\chi}\left(s_{\chi}+1\right)$ is normalized to unit for a Dirac fermion WIMP. By introducing the WIMP-nucleus form factor $F_{\chi-T}^{2}$, other SD operators can be generalized and expressed in a consistent form with Eq. (8) as

$$
\frac{d R}{d q}=N_{T} \frac{\rho_{\chi}}{m_{\chi}} \frac{16}{3} \frac{\sigma}{2 \mu_{p}^{2}} q \int_{v_{\min }}^{v_{e}} F_{\chi-T}^{2}\left(q / q_{0}, v / v_{0}, a_{p} / a_{n}\right) \frac{f(\mathbf{v})}{v} d^{3} v
$$

Two reference parameters $q_{0}=100 \mathrm{MeV}$, the typical scale of the momentum transferred in common direct detection experiments, and $v_{0}=220 \mathrm{~km} / \mathrm{s}$, the WIMP velocity dispersion, are brought in to keep $F_{\chi-T}^{2}$ a dimensionless factor. The nominal "cross section" $\sigma$ here is only a parameter that encodes the coupling strength $a$. The mass difference between proton and neutron is ignored in this work. In Table I, we also normalize these operators to dimensionless ones with $q_{0}$ and $v_{0}$, in line with the conventional SD operator $\mathcal{O}_{4}$, and absorb the relevant couplings $\left\{a_{N}\right\}$ into parameter $\sigma$. In this paper, we also consider a light mediator scenario in which the WIMP-nucleon interaction is mediated by a low mass particle and in the zero mass limit a $1 / q^{2}$ factor arising from the massless propagator appears as the coefficient of each operator in Eq. (5) . All these six SD operators and their counterparts with light mediator are summarized in Table I. For the purpose of illustration we take $\mathcal{O}=\sum a_{N} \mathcal{O}_{9, N}=a_{p} i \mathbf{S}_{\chi} \cdot\left(\mathbf{S}_{p} \times \mathbf{q}\right)+a_{n} i \mathbf{S}_{\chi} \cdot\left(\mathbf{S}_{n} \times \mathbf{q}\right)$ as an example, with equal couplings $a=a_{p}=a_{n}$. From Table \ we have: 


\begin{tabular}{|c|c|c|c|}
\hline operator & normalized operator & parameter $(\sigma)$ & $F_{\chi-T}^{2}$ \\
\hline \hline $\mathcal{O}_{3, N}$ & $\mathcal{O}_{3, N} /\left(q_{0} v_{0}\right)$ & $f a^{2} q_{0}^{2} v_{0}^{2}$ & $\sum \frac{a_{N} a_{N^{\prime}} F_{3,3}^{\left(N, N^{\prime}\right)} /\left(q_{0}^{2} v_{0}^{2}\right)}{a^{2}}$ \\
\hline $\mathcal{O}_{3, N} / q^{2}$ & $\mathcal{O}_{3, N}\left(q_{0} / q^{2} v_{0}\right)$ & $f a^{2} v_{0}^{2} / q_{0}^{2}$ & $\sum \frac{a_{N} a_{N^{\prime}}}{a^{2}} F_{3,3}^{\left(N, N^{\prime}\right)}\left(q_{0}^{2} / v_{0}^{2}\right)$ \\
\hline $\mathcal{O}_{4, N}$ & $\mathcal{O}_{4, N}$ & $f C\left(s_{\chi}\right) a^{2}$ & $\sum \frac{a_{N} a_{N^{\prime}}}{a^{2}} C^{-1}\left(s_{\chi}\right) F_{4,4}^{\left(N, N^{\prime}\right)}$ \\
\hline $\mathcal{O}_{4, N} / q^{2}$ & $\mathcal{O}_{4, N}\left(q_{0}^{2} / q^{2}\right)$ & $f C\left(s_{\chi}\right) a^{2} / q_{0}^{2}$ & $\sum \frac{a_{N} a_{N^{\prime}}}{a^{2}} C^{-1}\left(s_{\chi}\right) F_{4,4}^{\left(N, N^{\prime}\right)}\left(q_{0}^{4} / q^{4}\right)$ \\
\hline $\mathcal{O}_{6, N}$ & $\mathcal{O}_{6, N} / q_{0}^{2}$ & $f C\left(s_{\chi}\right) a^{2} q_{0}^{4}$ & $\sum \frac{a_{N^{\prime}} a_{N^{\prime}}}{a^{2}} C^{-1}\left(s_{\chi}\right) F_{6,6}^{\left(N, N^{\prime}\right)} / q_{0}^{4}$ \\
\hline $\mathcal{O}_{6, N} / q^{2}$ & $\mathcal{O}_{6, N} / q^{2}$ & $f C\left(s_{\chi}\right) a^{2}$ & $\sum \frac{a_{N} a_{N^{\prime}}}{a^{2}} C^{-1}\left(s_{\chi}\right) F_{6,6}^{\left(N, N^{\prime}\right)} / q^{4}$ \\
\hline $\mathcal{O}_{7, N}$ & $\mathcal{O}_{7, N} / v_{0}$ & $f a^{2} v_{0}^{2}$ & $\sum \frac{a_{N} a_{N^{\prime}}}{a^{2}} F_{7,7}^{\left(N, N^{\prime}\right)} / v_{0}^{2}$ \\
\hline $\mathcal{O}_{7, N} / q^{2}$ & $\mathcal{O}_{7, N} q_{0}^{2} /\left(v_{0} q^{2}\right)$ & $f a^{2} v_{0}^{2} / q_{0}^{4}$ & $\sum \frac{a_{N} a_{N^{\prime}}}{a^{2}} F_{7,7}^{\left(N, N^{\prime}\right)}\left(q_{0}^{4} / v_{0}^{2} q^{4}\right)$ \\
\hline $\mathcal{O}_{9, N}$ & $\mathcal{O}_{9, N} / q_{0}$ & $f C\left(s_{\chi}\right) a^{2} q_{0}^{2}$ & $\sum \frac{a_{N} a_{N^{\prime}}}{a^{2}} C^{-1}\left(s_{\chi}\right) F_{9,9}^{\left(N, N^{\prime}\right)} / q_{0}^{2}$ \\
\hline $\mathcal{O}_{9, N} / q^{2}$ & $\mathcal{O}_{9, N}\left(q_{0} / q^{2}\right)$ & $f C\left(s_{\chi}\right) a^{2} / q_{0}^{2}$ & $\sum \frac{a_{N} a_{N^{\prime}}}{a^{2}} C^{-1}\left(s_{\chi}\right) F_{9,9}^{\left(N, N^{\prime}\right)}\left(q_{0}^{2} / q^{4}\right)$ \\
\hline $\mathcal{O}_{10, N}$ & $\mathcal{O}_{10, N} / q_{0}$ & $f a^{2} q_{0}^{2}$ & $\sum \frac{a_{N} a_{N^{\prime}}}{a^{2}} 4 C^{-1}\left(s_{\chi}\right) F_{6,6}^{\left(N, N^{\prime}\right)} /\left(q^{2} q_{0}^{2}\right)$ \\
\hline $\mathcal{O}_{10, N} / q^{2}$ & $\mathcal{O}_{10, N}\left(q_{0} / q^{2}\right)$ & $f a^{2} / q_{0}^{2}$ & $\sum \frac{a_{N} a_{N^{\prime}}}{a^{2}} 4 C^{-1}\left(s_{\chi}\right) F_{6,6}^{\left(N, N^{\prime}\right)}\left(q_{0}^{2} / q^{6}\right)$ \\
\hline
\end{tabular}

Table I: The SD operators $\left\{\mathcal{O}_{i}\right\}(i=3,4,6,7,9,10)$ together with corresponding amplitudes and form factors $F_{\chi-T}^{2} \cdot\left\{F_{i, i}^{\left(N, N^{\prime}\right)}\right\}$ are defined in Ref. [20]. The summations are taken over proton and neutron, $N, N^{\prime}=p, n$. Three choices of parameter $a=a_{p}\left(a_{n}=0\right), a=a_{n}\left(a_{p}=0\right)$ and $a=a_{p}=$ $a_{n}\left(a_{p}=a_{n}\right)$ correspond to the three situations explained in the text. The normalized operators are listed in the second column and $f=\frac{3}{16} \frac{\mu_{p}^{2}}{\pi}$.

$$
F_{\chi-T}^{2}=\sum_{N, N^{\prime}=p, n} C^{-1}\left(s_{\chi}\right) F_{9,9}^{\left(N, N^{\prime}\right)} / q_{0}^{2}
$$

and

$$
\frac{\sigma}{2 \mu_{p}^{2}}=C\left(s_{\chi}\right) \frac{a^{2}}{2 \pi} \frac{3 q_{0}^{2}}{16} .
$$

$\left\{F_{i, i}^{\left(N, N^{\prime}\right)}\right\}$ are defined in Ref. [20].

\section{Direct detection constraints}

In this section, we try to study the constraints imposed by direct detection experiments on couplings of the effective SD operators in Table \ for elastic scattering, where the corresponding form factors are taken from Ref. [20]. In order to interpret such constraints in a uniform way, we translate the experimental bounds into the constraints on the effective cross section parameter $\sigma$ through Eq. (10), and to show the implications of ratio $a_{p} / a_{n}$ in our analysis, we consider the following three extreme situations for reference: $a_{n}=0, a_{p}=0$, 
and $a_{p}=a_{n}$.
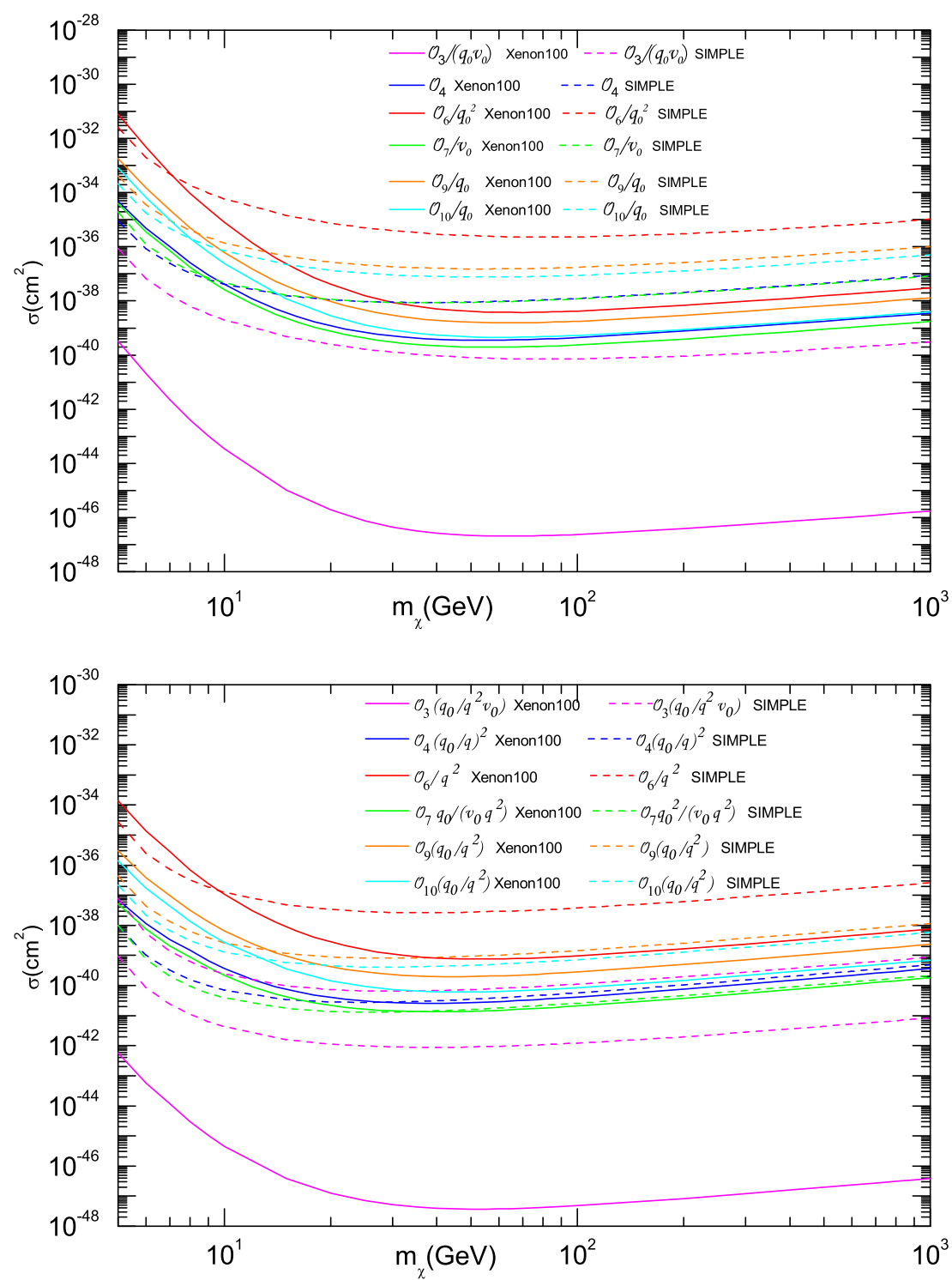

Figure 1: We combine the SIMPLE and Xenon100 90\% C.L. constraints on $\sigma$ for the normalized operators listed in Table I for elastic scattering, which are respectively the most sensitive in the low and high WIMP mass ranges, shown separately in two panels for the purpose of clear illustration, under the assumption $a_{p}=a_{n}$. 

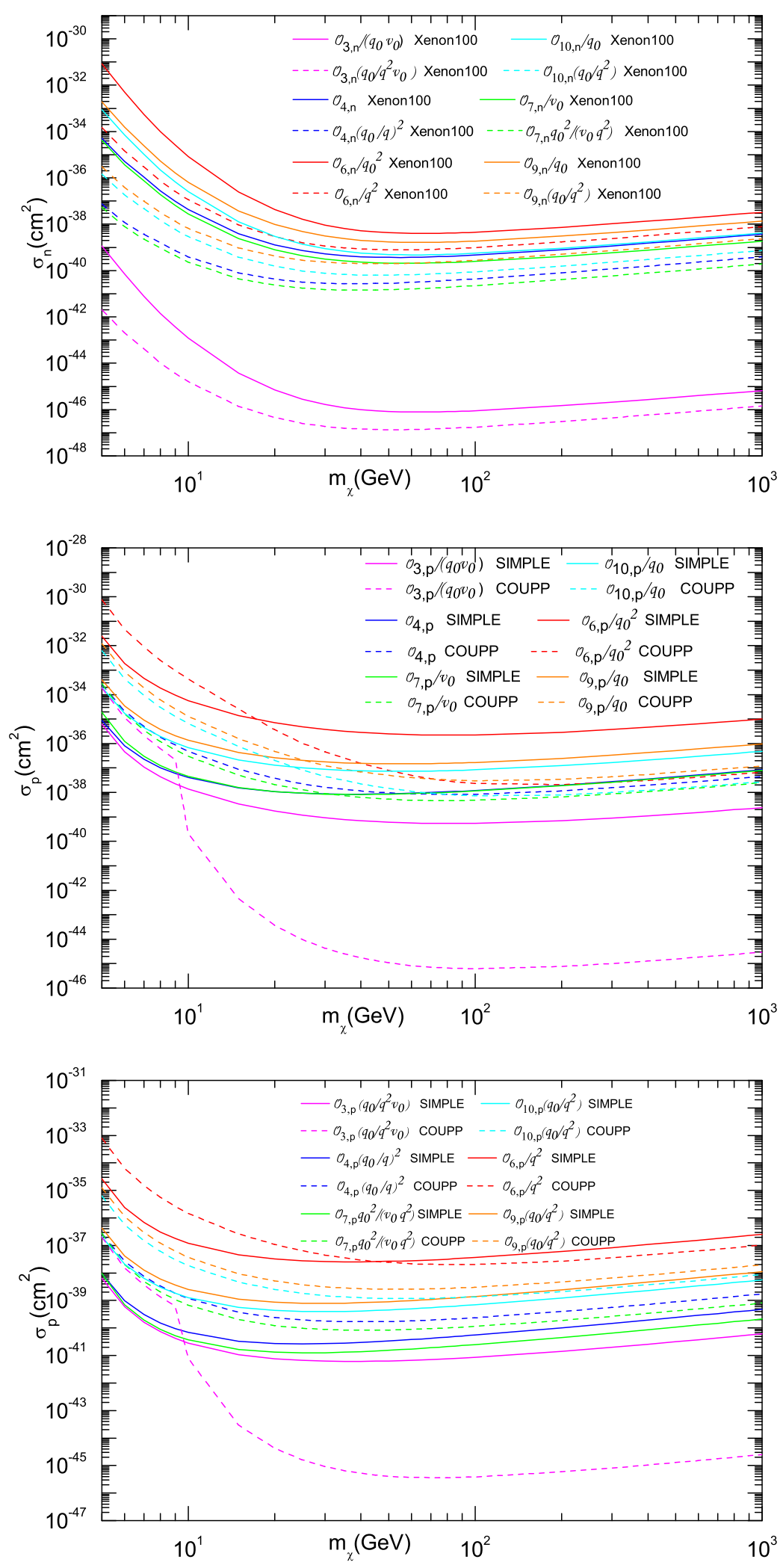

Figure 2: Xenon100 90\% C.L. upper limit on $\sigma_{n}$ for the normalized operators in Table \for elastic scattering assuming $a_{p}=0$ (top panel), and 90\% C.L. constraints on $\sigma_{p}$ from SIMPLE and COUPP for the case $a_{n}=0$ (middle and bottom panels) from Table \. 
Due to the unpaired proton in fluorine, and iodine, SIMPLE and COUPP are expected to possess a high efficiency in exploring the parameter space for the case $a_{n}=0$. If the WIMP couples exclusively to the neutron, we can use Xenon100 to place constraints by taking advantage of the odd number of neutrons in Xenon isotopes. As for the case of equal couplings $a_{p}=a_{n}$, comparisons between bounds from Xenon100 (neutron sensitive) and SIMPLE (proton sensitive) are also made in Fig. 1.

We use the reanalyzed data from the first stage of the phase II SIMPLE dark matter search [33], of which 5 out of 14 previous candidate events are attributed to background, reducing the expected signal rate to $0.289 /$ events $/ \mathrm{kgd}$ at $90 \%$ C.L. A more elaborate bubble nucleation efficiency $\eta=1-\exp \left[-\Gamma\left(E_{R} / E_{t h}-1\right)\right]$ is chosen, with $\Gamma=4.3 \pm 0.3$ and the threshold energy $E_{t h}=8 \mathrm{keV}$.

For COUPP [34], we adopt a similar exponential efficiency $\eta_{\mathrm{C}, \mathrm{F}}=1-\exp \left[-\alpha\left(E_{R} / E_{t h}-1\right)\right]$ with $\alpha=0.15$ for fluorine and carbon, and $\eta_{\mathrm{I}}=1$ for iodine above the nucleation threshold. We obtain 90\% C.L. exclusion contours by Poisson statistics with 13 observed events against the expected 4.5 background events [35]. Here we note that for the COUPP experiment we adopt the exponential bubble nucleation efficiency, which results in a more conservative exclusion limit when compared to the flat model.

For Xenon100, we follow [36, 37] to derive the 90\% C.L. limit curves by using the maximum gap method [38], under the assumption that the expected S1 signals are subject to the Poisson fluctuation, and taking into account the finite photomultiplier resolution $\sigma_{\mathrm{PMT}}=0.5 \mathrm{PE}$ (photon electron). Throughout our study the dark matter is assumed to be distributed in an isothermal halo with a local density $\rho_{\chi}=0.3 \mathrm{GeV} / \mathrm{cm}^{3}$ and a Maxwellian velocity distribution with a dispersion $v_{0}=220 \mathrm{~km} / \mathrm{s}$, truncated at the galactic escape velocity $v_{\mathrm{esc}}=544 \mathrm{~km} / \mathrm{s}$. We present the constraints for a Dirac fermionic WIMP in Fig. 1 and Fig. 2

In Fig. 1 we show the 90\% C.L. exclusion limits on $\sigma$ for the normalized operators listed in Table \for elastic scattering in the case of $a_{p}=a_{n}$. SIMPLE and Xenon100 are the most sensitive in the low and high WIMP mass ranges, respectively. The $90 \%$ C.L. exclusion contours on $\sigma_{n}\left(a_{p}=0\right)$ and $\sigma_{p}\left(a_{n}=0\right)$ are shown in Fig. 2, As discussed in Ref. [20, 21], the nuclear response of operator $\mathcal{O}_{3}\left(\mathcal{O}_{3} / q^{2}\right)$ may tend to favor a heavy element and hence bear a similarity to the standard SI response. One notes that the limit contour of operator $\mathcal{O}_{3}\left(\mathcal{O}_{3} / q^{2}\right)$ features a sharp decline around $10 \mathrm{GeV}$, where the response of the heavy element 
iodine is suddenly switched on due to the step function of the nucleation efficiency.

\section{DARK MATTER CAPTURE BY SUN}

\section{A. Capture rate and annihilation rate}

The capture of dark matter takes place when the incoming WIMPs collide with the solar

elements and are stripped of enough kinetic energy to escape from the gravitational pull of the Sun. We calculate this process in the Sun's rest frame, in which the WIMP velocity distribution is written as

$$
f(\mathbf{u})=\frac{e^{-\frac{\left(\mathbf{u}+\mathbf{v}_{\odot}\right)^{2}}{v_{0}^{2}}}}{N\left(v_{\mathrm{esc}}\right)},
$$

where $\mathbf{v}_{\odot}$ is the velocity of the Sun and $\mathbf{u}$ is the DM velocity at infinity with respect to the Sun's rest frame, $N\left(v_{\text {esc }}\right)$ being the normalization constant dependent on $v_{\text {esc }}$. Due to the smallness of the WIMP-nucleus cross section, the Sun is assumed to be optically thin to the incoming WIMPs and hence multiple scatterings are neglected. By use of the DM angular momentum conservation in the solar central field, one can obtain the following WIMP scattering event rate $R_{\odot}[39]$,

$$
R_{\odot}=\sum_{A_{i}} \int_{\text {Sun }} d V \int \frac{f(\mathbf{u})}{u} w \Omega_{A_{i}}^{-}(w) d^{3} u,
$$

in which the summation is taken over all elements $\left\{A_{i}\right\}$ in the Sun. $\Omega_{A_{i}}^{-}(w)$ is defined as

$$
\Omega_{A_{i}}^{-}(w)=n_{A_{i}}(r) n_{\chi} \sigma(w) w
$$

$n_{A_{i}}(r)$ is the number density of element $A_{i}$ at radius $r$ and the local WIMP density $n_{\chi}$ in the solar neighborhood is determined by $n_{\chi}=\rho_{\chi} / m_{\chi} \cdot w(r)=\sqrt{u^{2}+v_{\mathrm{esc}}^{2}(r)}$ is the incident DM velocity at radius $r$ inside the Sun, accelerated from the initial velocity $u$ at infinity by the solar gravitational attraction. $v_{\text {esc }}(r)$, the escape velocity at radius $r$, is related to that at the Sun's center $v_{c}=1354 \mathrm{~km} / \mathrm{s}$ and at surface $v_{s}=795 \mathrm{~km} / \mathrm{s}$ by the following approximate relation [40]: 


$$
v_{\mathrm{esc}}^{2}(r)=v_{c}^{2}-\frac{M(r)}{M_{\odot}}\left(v_{c}^{2}-v_{s}^{2}\right) .
$$

$M_{\odot}$ is the mass of the Sun and $M(r)$ is the mass contained within radius $r$. Since only those WIMPs that lose enough energy after scattering can be trapped by the Sun, for the solar capture it is then demanded that the scattered WIMPs be contained in the radius of Jupiter's orbit $r_{0}$ [5, 41], which implies

$$
\frac{q^{2}}{2 m_{A}} \geq \frac{m_{\chi} u^{2}}{2}+\frac{m_{\chi} v_{\mathrm{esc}}^{2}\left(r_{0}\right)}{2}
$$

with $v_{\text {esc }}\left(r_{0}\right)=18.5 \mathrm{~km} / \mathrm{s}$. Therefore, to derive the capture rate $C_{\odot}$, one can replace $\sigma(w)$ in Eq. (15) with an effective capture cross section,

$$
\sigma_{\mathrm{SD}, A_{i}}^{\mathrm{cap}}(w)=\frac{16}{3} \frac{\sigma}{2 \mu_{p}^{2} w^{2}} \int_{q_{\min }}^{2 \mu_{A_{i}} w} F_{\chi-T}^{2}(q, w) q d q,
$$

where $q_{\text {min }}$ is determined from Eq. (17). Then we have

$$
C_{\odot}=\sum_{A_{i}} \int_{\text {Sun }} d V \int \frac{f(\mathbf{u})}{u} w \Omega_{\text {cap }, A_{i}}^{-}(w) d^{3} u
$$

with

$$
\Omega_{c a p, A_{i}}^{-}=n_{A_{i}}(r) n_{\chi} \sigma_{\mathrm{SD}, A_{i}}^{\mathrm{cap}}(w) w
$$

One should note that besides depicting the motion of the WIMPs in a more realistic three-body interaction picture, the introduction of such a finite radius $r_{0}$ simultaneously avoids the divergence disaster that we encounter in the calculation of capture rate in the massless mediator scenario. So it is reasonable to expect that a massless force carrier will give a good representative description of one with a low but finite mass for capture.

Moreover, if we make the approximation by setting $v_{\text {esc }} \rightarrow \infty$, the expression of differential capture rate can be simplified remarkably to

$$
\frac{d C_{\odot}}{d V}=\int_{0}^{\infty} \frac{4 \pi u^{2} d u}{\left(\pi v_{0}^{2}\right)^{3 / 2}} \frac{v_{0}^{2}}{2 u v_{\odot}} \Omega_{\text {cap }, A_{i}}^{-}(w) \frac{w}{u} \exp \left[-\left(\frac{u^{2}+v_{\odot}^{2}}{v_{0}^{2}}\right)\right] \sinh \left(\frac{2 u v_{\odot}}{v_{0}^{2}}\right) .
$$

As we merely focus on the spin-dependent operators in this paper, only contribution of hydrogen atoms to the capture rate is relevant in our calculation. This is because all the 
SD operators in Table $\llbracket$ except $\mathcal{O}_{3}$ and $\mathcal{O}_{3} / q^{2}$ lead to amplitudes proportional to the spin or angular momentum of the nucleus at small $q$, rather than the square of atomic number $A_{i}$. It indicates that the contribution from heavy elements are subject to a significant abundance-suppression. As for $\mathcal{O}_{3}$ and $\mathcal{O}_{3} / q^{2}$, however, the amplitudes may grow with atomic number [20], it then requires a thorough understanding about the relevant nuclear structure of those heavy elements present in the Sun, which is beyond the scope of this work. The distributions of the elements are obtained from the Standard Sun Model (SSM) GS98 [42].

Under the assumption of a large WIMP mean free path, the trapped WIMPs thermalize and sink into the core of the Sun, so the WIMP annihilation takes place at the center region , depleting the WIMP population through annihilation and evaporation. The evolution of the WIMP number $N$ in the Sun is described by the following equation,

$$
\dot{N}=C_{\odot}-A_{\odot} N^{2}-E_{\odot} N
$$

which includes the effects of capture $\left(C_{\odot}\right)$, annihilation $\left(A_{\odot}\right)$, and evaporation $\left(E_{\odot}\right)$. The annihilation rate $A_{\odot}$ is defined as

$$
A_{\odot}=\frac{\langle\sigma v\rangle_{\odot}}{V_{\mathrm{eff}}}
$$

where $\langle\sigma v\rangle_{\odot}$ is the thermal average over the annihilation cross section times the relative velocity and $V_{\text {eff }}$ is the effective volume for annihilation, which can be approximately given as [39, 43]

$$
V_{\text {eff }}=5.8 \times 10^{30} \mathrm{~cm}^{3}\left(\frac{1 \mathrm{GeV}}{m_{\chi}}\right)^{3 / 2} .
$$

The evaporation mass $m_{e v}$ is defined as a characteristic parameter above which the WIMP evaporation effect is negligible. As an estimate the evaporation mass for a SD cross section $\sigma_{\mathrm{SD}} \sim 4 \times 10^{-36} \mathrm{~cm}^{2}$ is about $3 \mathrm{GeV}$ [4]], so we neglect the evaporation effect in the WIMP mass range of our interest $\left(m_{\chi} \geqslant 5 \mathrm{GeV}\right)$, considering $m_{e v}$ depends on the WIMP-hydrogen cross section in a logarithmic manner for the rare scattering scenario [43-45]. Thus, one can easily obtain the solution to Eq.(22) as 


$$
N(t)=\sqrt{\frac{C_{\odot}}{A_{\odot}}} \tanh \left(\sqrt{C_{\odot} A_{\odot}} t\right),
$$

so the present annihilation rate can be immediately written as

$$
\Gamma_{\odot}=\frac{1}{2} A_{\odot} N^{2}\left(t_{\odot}\right)=\frac{1}{2} C_{\odot} \tanh ^{2}\left(\sqrt{C_{\odot} A_{\odot}} t_{\odot}\right) .
$$

$t_{\odot} \simeq 4.5 \times 10^{9} \mathrm{yr}$ is the age of the Sun. If $\sqrt{C_{\odot} A_{\odot}} t_{\odot} \gg 1$, the DM capture-annihilation process reaches equilibrium, and as a result the annihilation rate is solely determined by capture rate through $\Gamma_{\odot}=\frac{1}{2} C_{\odot}$.

\section{B. Constraints from Super-Kamionkande and IceCube}

The differential flux of muon neutrino observed at the Earth for annihilation channel $f$ is

$$
\frac{d \Phi_{\nu_{\mu}}^{f}}{d E_{\nu_{\mu}}}=\frac{\Gamma_{\odot}}{4 \pi d^{2}} \frac{d N_{\nu_{\mu}}^{f}}{d E_{\nu_{\mu}}},
$$

where $d$ is the Earth-Sun distance, and $d N_{\nu_{\mu}}^{f} / d E_{\nu_{\mu}}$ is the differential energy spectrum of the muon neutrino. In order to fully and accurately determine the neutrino spectrum, a wide variety of phenomena must be taken into consideration including the hadronization of quarks, neutrino oscillations, energy loss in the solar medium and en route to the Earth, etc. Here we choose the following neutrino oscillation parameters [46, 47]:

$$
\begin{gathered}
\sin ^{2} \theta_{12}=0.32, \quad \sin ^{2} \theta_{23}=0.49, \quad \sin ^{2} \theta_{13}=0.026, \quad \delta=0.83 \pi \\
\Delta m_{21}^{2}=7.62 \times 10^{-5} \mathrm{eV}^{2}, \quad \Delta m_{31}^{2}=2.53 \times 10^{-3} \mathrm{eV}^{2}
\end{gathered}
$$

The upgoing muons produced from the interactions between the arriving neutrinos and the Earth rocks or ice can be detected by the water Cherenkov detector SuperKamionkande [28] and the neutrino telescope IceCube [29, 30], then one can map the upper limits on the muon flux into the constraints on the WIMP annihilation rate by assuming specific annihilation modes and considering relevant details in neutrino propagation. 
For Super-Kamionkande the neutrino-induced muon events are divided into three categories: fully-contained, stopping and through-going, and the fraction of each category as a function of the parent neutrino energy $E_{\nu_{\mu}}$ is shown in Fig. 2 in Ref. [28]. On the other hand, the IceCube Collaboration has also reported the constraints on the DM annihilation rate $\Gamma_{\odot}$ for $\bar{b} b, W^{+} W^{-}$, and $\tau^{+} \tau^{-}\left(m_{\chi}<80.4 \mathrm{GeV}\right)$ channels in Table I of Ref. [29], together with the expected 180-day sensitivity of the completed IceCube detector . Here we adopt the relevant detection parameters summarized in Table II and the $90 \%$ C. L. upper limits on the annihilation rates from Ref. [31]. In order to show how the equilibrium assumption remains a good approximation, we take the criterion that $\sqrt{C_{\odot} A_{\odot}} t_{\odot} \geq 3.0\left(\tanh ^{2}\left(\sqrt{C_{\odot} A_{\odot}} t_{\odot}\right) \geq 0.99\right)$ or $C_{\odot} / 2 \geq 4.3 \times 10^{22}\left(1 \mathrm{GeV} / m_{\chi}\right)^{3 / 2} \mathrm{~s}^{-1}$ for the $s$-wave thermally averaged annihilation cross section $\langle\sigma v\rangle_{\odot} \approx 3.0 \times 10^{-26} \mathrm{~cm}^{3} \mathrm{~s}^{-1}$, which is plotted in a black solid line in Fig. 3 and Fig. 4. We can see that the constraints deduced from the neutrino detectors are far above the equilibrium contour, which assures the equilibrium assumption $\Gamma_{\odot}=\frac{1}{2} C_{\odot}$.

Meanwhile we translate the above-acquired constraints on the elastic WIMP-proton coupling $a_{p}$ into the bounds on the annihilation rate $\Gamma_{\odot}$ by involving only hydrogen presence in our calculation. All these results are shown in Fig. 3 and Fig. 4. To project the upper limits on the elastic WIMP-nucleon couplings onto the annihilation rate, we only need to replace the parameter $\sigma$ and the WIMP-nucleus form factor $F_{\chi-T}^{2}$ in Eq.(18) with the corresponding upper limit $\sigma^{\text {limit }}\left(a^{\text {limit }}\right)$ drawn from direct detections and the WIMP-hydrogen form factor. For instance, the constraint on the effective capture cross section for operator $\mathcal{O}_{9, p}=i \mathbf{S}_{\chi} \cdot\left(\mathbf{S}_{p} \times \mathbf{q}\right)$ can be obtained as the following,

$$
\begin{aligned}
\sigma_{\mathrm{SD}, \mathcal{O}_{9}, p}^{\text {cap } \text { limit }_{i}}(w) & =\frac{\left(a_{p}^{\text {limit }}\right)^{2}}{2 \pi w^{2}} \frac{1}{\left(2 s_{p}+1\right)\left(2 s_{\chi}+1\right)} \sum_{\text {spins }} \int_{q_{\text {min }}}^{2 \mu_{\mathrm{p}} w}\left|\left\langle i \mathbf{S}_{\chi} \cdot\left(\mathbf{S}_{p} \times \mathbf{q}\right)\right\rangle\right|^{2} q d q \\
& =\frac{2}{3} \frac{\sigma_{\mathcal{O}_{9}, p}^{\text {limit }}}{2 \mu_{p}^{2} w^{2}} \int_{q_{\min }}^{2 \mu_{\mathrm{p}} w}\left(\frac{q}{q_{0}}\right)^{2} q d q,
\end{aligned}
$$

where $\mathbf{S}_{p}\left(s_{p}\right)$ represents the spin of the hydrogen atom (proton). 

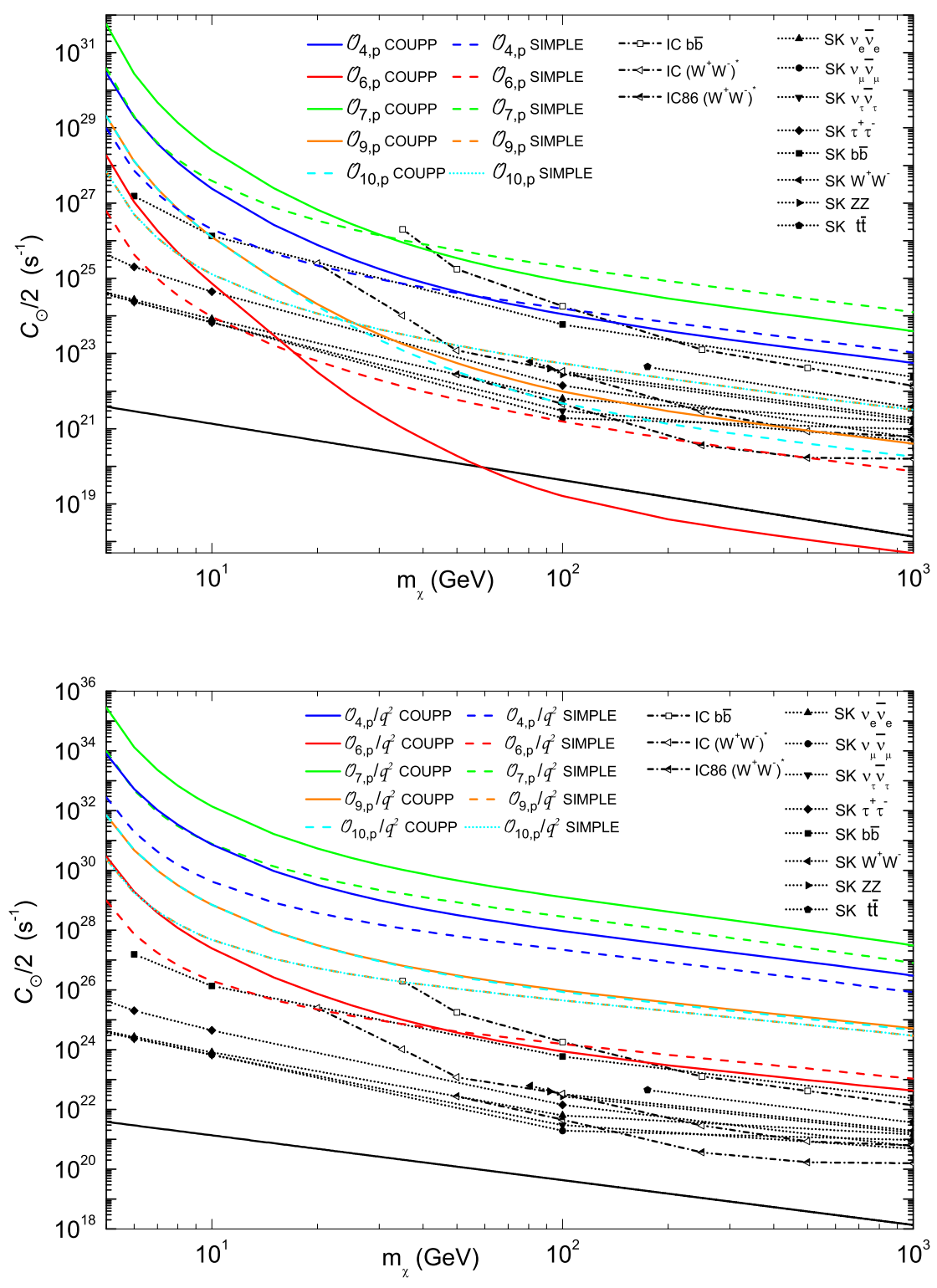

Figure 3: the $90 \%$ C.L. upper limits on $C_{\odot} / 2$ for various Dirac fermionic WIMP SD operators for elastic scattering in Table \in the case $a_{n}=0$, under the assumption that the equilibrium between the WIMP capture and annihilation is reached. The black line denotes the equilibrium criterion detailed in the text. To clearly illustrate the results, the constraints are shown in two separate panels. 

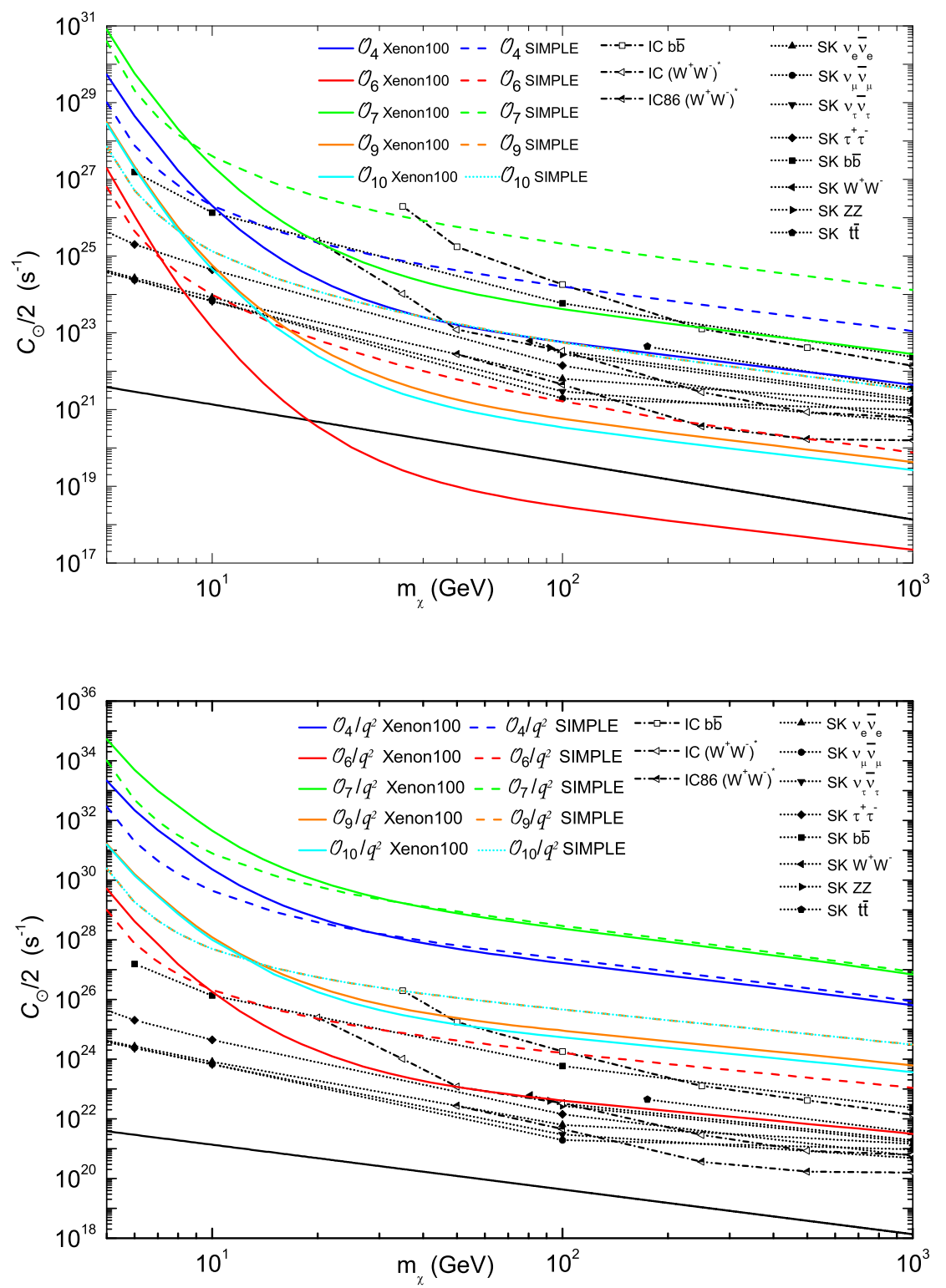

Figure 4: the $90 \%$ C.L. upper limits on $C_{\odot} / 2$ for various SD operators for elastic scattering in Table \ in the case $a_{n}=a_{p}$, under the assumption that the equilibrium between the DM capture and annihilation is reached. The black line denotes the equilibrium criterion detailed in the text. To clearly illustrate the results, the constraints are shown in two separate panels.

We can also derive the upper limit on the effective capture cross section for operator $\mathcal{O}_{10, p}=i \mathbf{S}_{p} \cdot \mathbf{q}$ in a similar way,

$$
\sigma_{\mathrm{SD}, \mathcal{O}_{10}, p}^{\text {cap }, \text { limit }}(w)=\frac{4}{3} \frac{\sigma_{\mathcal{O}_{10}, p}^{\text {limit }}}{2 \mu_{p}^{2} w^{2}} \int_{q_{\min }}^{2 \mu_{\mathrm{p}} w}\left(\frac{q}{q_{0}}\right)^{2} q d q .
$$

It is worth noting that unlike the case of direct detection, in which $\sigma$ depends on the 
normalization parameters $q_{0}$ and $v_{0}$, the constraints on the annihilation rate (or capture rate) merely depend on the operators in the first column in Table @, as shown in the first line of Eq. (29).

One can learn from Fig. 3 that when the proton-coupling dominant $\operatorname{scenario}\left(a_{n}=0\right)$ is assumed, the Super-Kamionkande provides more stringent constraints on SD operators $\mathcal{O}_{4}$ and $\mathcal{O}_{7}$ than the direct detection experiments SIMPLE and COUPP do. However, for $\mathcal{O}_{6}$ both SIMPLE and COUPP become more sensitive for the DM mass range $m_{\chi}>20 \mathrm{GeV}$. For the light mediator case, two neutrino detectors are proved to be more effective in exploring the parameter space. Similar situations can be found in Fig. 4 for the equal coupling scenario $\left(a_{p}=a_{n}\right)$. On the other hand, for the simplest SD interaction $\mathcal{O}_{4}$ with only WIMPproton coupling shown in the top panel in Fig. 3, the neutrino-based constraints lead in the detection sensitivity over that of the direct detection approach by a factor up to $2 \sim 3$ orders of magnitude, depending on the specific annihilation channels. However, with the power of the transferred momentum $q$ increased in the effective operator, the direct detections turn more effective in excluding the parameter space, especially in the large WIMP mass region that favors a large transferred momentum. For $\mathcal{O}_{6} \propto q^{2}$, both constraints from SIMPLE and COUPP reach below that of the indirect search Super-Kamionkande and IceCube in the region $m_{\chi}>20 \mathrm{GeV}$. For the same reason, considering a propagator inversely proportional to $q^{2}$, one would expect that the bounds tend to go upwards in the massless mediator scenario in the bottom panel of Fig. 3. Similar arguments still hold for the equal coupling case, where the stringent constraints on the couplings in direct detection experiments can also be obtained by Xenon100, mainly through the WIMP-neutron interaction.

It is noted that the constraints on the annihilation rates of $\mathcal{O}_{9, p}$ and $\mathcal{O}_{10, p}$ are coincident with each other in Fig. 3. To explain this coincidence, we first compare the two upper limits $\sigma_{p, 9}^{\text {limit }}$ and $\sigma_{p, 10}^{\text {limit }}$ from Table $\llbracket$ and find the following relation:

$$
\frac{\sigma_{\mathcal{O}_{10}, p}^{\text {limit }}}{\sigma_{\mathcal{O}_{9}, p}^{\operatorname{limit}}}=\frac{1}{4} \frac{F_{\Sigma^{\prime}}^{(p, p)}}{F_{\Sigma^{\prime \prime}}^{(p, p)}} .
$$

So in light of Eq. (29) and Eq. (30) we further have

$$
\frac{C_{\odot, \mathcal{O}_{10}, p}^{\text {limit }}}{C_{\odot, \mathcal{O}_{9}, p}^{\text {limit }}}=\frac{1}{2} \frac{F_{\Sigma^{\prime}}^{(p, p)}}{F_{\Sigma^{\prime \prime}}^{(p, p)}},
$$


where $C_{\odot, \mathcal{O}_{9, p}}^{\text {limit }}$ and $C_{\odot, \mathcal{O}_{10}, p}^{\text {limit }}$ are the relevant upper limits on the capture rate of $\mathcal{O}_{9, p}$ and $\mathcal{O}_{10, p}$,

respectively. Since the ratio between the transverse form factor $F_{\Sigma^{\prime}}^{(p, p)}$ and the longitudinal one $F_{\Sigma^{\prime \prime}}^{(p, p)}$ approaches 2 in the long-wavelength limit and fluorine favors a small transferred momentum in direct detection, one can understand why the constraints on the annihilation rates of $\mathcal{O}_{9, p}$ and $\mathcal{O}_{10, p}$ are inseparable in Fig. 3. Similar arguments can be applied to explain the coincidence of $\mathcal{O}_{9}$ and $\mathcal{O}_{10}$ in Fig. [4.

\section{DISCUSSION AND CONCLUSION}

In this paper we have studied how a diversity of effective SD operators can lead to different interpretations of some direct detection experimental results, based on the nuclear form factors given in [20]. For each type of operator, we further group the possible interactions into three illustrative categories: $a_{n}=0, a_{p}=0$, and $a_{p}=a_{n}$. When the WIMP couples dominantly with proton (neutron) over neutron (proton), the first (second) category lives up to a good approximation, and when the two coupling strengths are comparable, we expect the third category $a_{p}=a_{n}$ to give a representative description. We have used protonsensitive experiments SIMPLE and COUPP to plot the upper limits on the proton coupling $\left(\sigma_{p}\right)$ and used neutron-sensitive experiment Xenon100 to constrain the neutron coupling $\left(\sigma_{n}\right)$. One can draw from Fig. 2 that SIMPLE is more effective in excluding parameter space below the WIMP mass of tens of GeV, whereas COUPP turns more strict in the larger WIMP mass range due to the heavy element iodine that favors a larger recoil energy. However, when the massless mediator scenario is involved in consideration, the propagator provides an enhancement in the low transferred momentum regime which makes SIMPLE more sensitive in the whole WIMP mass range. In Fig. 1 we have shown the $90 \% \mathrm{C}$. L. upper limits on the SD operators for the equal coupling scenario, in which one can see the Xenon100 and SIMPLE give complementary constraints on $\sigma$ in combination.

Since all those SD operators (except $\mathcal{O}_{3}$ and $\mathcal{O}_{3} / q^{2}$ ) listed in Table \result in scattering amplitudes proportional to the angular momentum or spin of the solar elements in the long-wavelength limit, unlike the SI amplitudes that are usually with an $A^{2}$ enhancement, we have only calculated the contribution of hydrogen to the WIMP capture rate. This is because other solar component elements related to the SD interactions are significantly suppressed due to their low abundance in the Sun. As for operator $\mathcal{O}_{3}\left(\right.$ or $\mathcal{O}_{3} / q^{2}$ ), the 
nuclear response is sensitive to $\sum_{i} \mathbf{L}_{i} \cdot \mathbf{S}_{i}$ which may favor heavier elements, so that we must take heavy elements into our consideration just as we did in the SI case. Unfortunately, so far there is no such knowledge of the nuclear form factors of the relevant elements, so we should leave this investigation to future work. We have also mapped the constraints on the WIMP-nucleon couplings onto the bounds on the annihilation rate deduced from the solar neutrino experiments, Super-Kamionkande and IceCube, under the equilibrium assumption, which allows us to provide complementary exclusion contours from both direct and indirect detection experiments.

Finally, we point out that our discussions concerning the WIMP capture and distribution

are based on the assumption of a large Knudsen number $(K>1)$ [44, 48]. In that picture, the WIMP's free path is much larger than the length of the WIMP populated region, and its distribution can be described as isothermal with a characteristic temperature $T_{\chi}$ [44]. Strictly speaking, the large Knudsen number assumption may no longer be valid for some nonconventional operators at the parameter $(\sigma)$ scales probed by the present direct detection experiments. For instance, in the case where the WIMP and nucleus interact through a light force carrier, the relevant cross section for WIMPs that reside in the Sun's core will receive a boost factor relative to the one required for capture. If the boost factor is so large that the WIMPs and nuclei collide frequently in the inner part of the Sun, the interaction moves into the local thermal equilibrium region (LTE) [49], in which the WIMP distribution and energy transport are discussed recently by other authors in Ref. [50]. To study the solar DM signals for some unconventional operators in a more realistic way, we have to take the LTE scenario into consideration, which we leave to our future work.

\section{Acknowledgments}

We thank Wanlei Guo for useful discussions. This work is supported in part by the National Basic Research Program of China (973 Program) under Grant No. 2010CB833000, the National Nature Science Foundation of China (NSFC) under Grants No. 10821504 and No. 10905084, and the Project of Knowledge Innovation Program (PKIP) of the Chinese 
Academy of Science.

[1] G. Jungman, M. Kamionkowski, and K. Griest, Supersymmetric dark matter, Phys.Rept. 267 (1996) 195-373, hep-ph/9506380.

[2] G. Bertone, D. Hooper, and J. Silk, Particle dark matter: Evidence, candidates and constraints, Phys.Rept. 405 (2005) 279-390, [hep-ph/0404175].

[3] J. L. Feng, J. Kumar, D. Marfatia, and D. Sanford, Isospin-Violating Dark Matter, Phys.Lett. B703 (2011) 124-127, arXiv:1102.4331.

[4] T. Schwetz and J. Zupan, Dark Matter attempts for CoGeNT and DAMA, JCAP 1108 (2011) 008, arXiv:1106.6241.

[5] J. Kumar, J. G. Learned, S. Smith, and K. Richardson, Tools for Studying Low-Mass Dark Matter at Neutrino Detectors, Phys.Rev. D86 (2012) 073002, arXiv:1204.5120].

[6] D. Tucker-Smith and N. Weiner, Inelastic dark matter, Phys.Rev. D64 (2001) 043502, hep-ph/0101138.

[7] B. Feldstein, A. L. Fitzpatrick, and E. Katz, Form Factor Dark Matter, JCAP 1001 (2010) 020, arXiv:0908.2991.

[8] E. Masso, S. Mohanty, and S. Rao, Dipolar Dark Matter, Phys.Rev. D80 (2009) 036009, arXiv:0906.1979].

[9] V. Barger, W.-Y. Keung, and D. Marfatia, Electromagnetic properties of dark matter: Dipole moments and charge form factor, Phys.Lett. B696 (2011) 74-78, arXiv:1007.4345].

[10] S. Chang, N. Weiner, and I. Yavin, Magnetic Inelastic Dark Matter, Phys.Rev. D82 (2010) 125011, arXiv:1007.4200].

[11] A. L. Fitzpatrick and K. M. Zurek, Dark Moments and the DAMA-CoGeNT Puzzle, Phys.Rev. D82 (2010) 075004, arXiv:1007.5325].

[12] M. Farina, D. Pappadopulo, A. Strumia, and T. Volansky, Can CoGeNT and DAMA Modulations Be Due to Dark Matter?, JCAP 1111 (2011) 010, arXiv:1107.0715.

[13] N. Fornengo, P. Panci, and M. Regis, Long-Range Forces in Direct Dark Matter Searches, Phys.Rev. D84 (2011) 115002, arXiv:1108.4661.

[14] R. Foot, Mirror and hidden sector dark matter in the light of new CoGeNT data, Phys.Lett. B703 (2011) 7-13, arXiv:1106.2688. 
[15] M. T. Frandsen, F. Kahlhoefer, J. March-Russell, C. McCabe, M. McCullough, et al., On the DAMA and CoGeNT Modulations, Phys.Rev. D84 (2011) 041301, arXiv:1105.3734].

[16] E. Del Nobile, C. Kouvaris, P. Panci, F. Sannino, and J. Virkajarvi, Light Magnetic Dark Matter in Direct Detection Searches, JCAP 1208 (2012) 010, arXiv:1203.6652.

[17] U. Haisch and F. Kahlhoefer, On the importance of loop-induced spin-independent interactions for dark matter direct detection, JCAP 1304 (2013) 050, arXiv:1302.4454].

[18] M. T. Frandsen, F. Kahlhoefer, C. McCabe, S. Sarkar, and K. Schmidt-Hoberg, The unbearable lightness of being: CDMS versus XENON, JCAP 1307 (2013) 023, arXiv:1304.6066.

[19] J. Fan, M. Reece, and L.-T. Wang, Non-relativistic effective theory of dark matter direct detection, JCAP 1011 (2010) 042, arXiv:1008.1591.

[20] A. L. Fitzpatrick, W. Haxton, E. Katz, N. Lubbers, and Y. Xu, The Effective Field Theory of Dark Matter Direct Detection, JCAP 1302 (2013) 004, arXiv:1203.3542.

[21] A. L. Fitzpatrick, W. Haxton, E. Katz, N. Lubbers, and Y. Xu, Model Independent Direct Detection Analyses, arXiv:1211.2818.

[22] E. Del Nobile, M. Cirelli, and P. Panci, Tools for model-independent bounds in direct dark matter searches, arXiv:1307.5955.

[23] P. Klos, J. Menendez, D. Gazit, and A. Schwenk, Large-scale nuclear structure calculations for spin-dependent WIMP scattering with chiral effective field theory currents, arXiv:1304.7684.

[24] K. Belotsky, T. Damour, and M. Y. Khlopov, Implications of a solar system population of massive fourth generation neutrinos for underground searches of monochromatic neutrino annihilation signals, Phys.Lett. B529 (2002) 10-18, astro-ph/0201314.

[25] D. Hooper, F. Petriello, K. M. Zurek, and M. Kamionkowski, The New DAMA Dark-Matter Window and Energetic-Neutrino Searches, Phys.Rev. D79 (2009) 015010, arXiv:0808.2464].

[26] F. Halzen and D. Hooper, The Indirect Search for Dark Matter with IceCube, New J.Phys. 11 (2009) 105019, arXiv:0910.4513.

[27] R. Kappl and M. W. Winkler, New Limits on Dark Matter from Super-Kamiokande, Nucl.Phys. B850 (2011) 505-521, arXiv:1104.0679.

[28] Super-Kamiokande Collaboration, T. Tanaka et al., An Indirect Search for WIMPs in the Sun using 3109.6 days of upward-going muons in Super-Kamiokande, Astrophys.J. $\mathbf{7 4 2}$ 
(2011) 78, arXiv:1108.3384.

[29] IceCube collaboration, M. Aartsen et al., Search for dark matter annihilations in the Sun with the 79-string IceCube detector, Phys.Rev.Lett. 110 (2013) 131302, arXiv:1212.4097.

[30] IceCube Collaboration, R. Abbasi et al., Multi-year search for dark matter annihilations in the Sun with the AMANDA-II and IceCube detectors, Phys.Rev. D85 (2012) 042002, [arXiv:1112.1840].

[31] W.-L. Guo, Z.-L. Liang, and Y.-L. Wu, Direct detection and solar capture of dark matter with momentum and velocity dependent elastic scattering, arXiv:1305.0912,

[32] N. Anand, A. L. Fitzpatrick, and W. Haxton, Model-independent WIMP Scattering Responses and Event Rates: A Mathematica Package for Experimental Analysis, arXiv:1308.6288.

[33] M. Felizardo, T. Girard, T. Morlat, A. Fernandes, A. Ramos, et al., Final Analysis and Results of the Phase II SIMPLE Dark Matter Search, Phys.Rev.Lett. 108 (2012) 201302, arXiv:1106.3014].

[34] COUPP Collaboration, E. Behnke et al., First Dark Matter Search Results from a 4-kg $\mathrm{CF}_{3} I$ Bubble Chamber Operated in a Deep Underground Site, Phys.Rev. D86 (2012) 052001, arXiv:1204.3094.

[35] M. Cannoni, Reanalysis of nuclear spin matrix elements for dark matter spin-dependent scattering, Phys.Rev. D87 (2013) 075014, arXiv:1211.6050.

[36] XENON100 Collaboration, E. Aprile et al., Likelihood Approach to the First Dark Matter Results from XENON100, Phys.Rev. D84 (2011) 052003, arXiv:1103.0303.

[37] XENON100 Collaboration, E. Aprile et al., Limits on spin-dependent WIMP-nucleon cross sections from 225 live days of XENON100 data, arXiv:1301.6620.

[38] S. Yellin, Finding an upper limit in the presence of unknown background, Phys.Rev. D66 (2002) 032005, physics/0203002.

[39] A. Gould, Resonant Enhancements in WIMP Capture by the Earth, Astrophys.J. 321 (1987) 571.

[40] A. Gould, Cosmological density of WIMPs from solar and terrestrial annihilations, Astrophys.J. (1991).

[41] A. H. Peter, Dark matter in the solar system II: WIMP annihilation rates in the Sun, Phys.Rev. D79 (2009) 103532, arXiv:0902.1347.

[42] A. Serenelli, S. Basu, J. W. Ferguson, and M. Asplund, New Solar Composition: The 
Problem With Solar Models Revisited, Astrophys.J. 705 (2009) L123-L127, arXiv:0909.2668.

[43] K. Griest and D. Seckel, Cosmic Asymmetry, Neutrinos and the Sun, Nucl.Phys. B283 (1987) 681.

[44] D. Spergel and W. Press, Effect of hypothetical, weakly interacting, massive particles on energy transport in the solar interior, Astrophys.J. 294 (1985) 663-673.

[45] A. Gould, WIMP DISTRIBUTION IN AND EVAPORATION FROM THE SUN, Astrophys.J. 321 (1987) 560.

[46] DAYA-BAY Collaboration, F. An et al., Observation of electron-antineutrino disappearance at Daya Bay, Phys.Rev.Lett. 108 (2012) 171803, arXiv:1203.1669.

[47] D. Forero, M. Tortola, and J. Valle, Global status of neutrino oscillation parameters after Neutrino-2012, Phys.Rev. D86 (2012) 073012, arXiv:1205.4018.

[48] A. Gould and G. Raffelt, Cosmion Energy Transfer in Stars: The Knudsen Limit, Astrophys.J. 352 (1990) 669.

[49] A. Gould and G. Raffelt, THERMAL CONDUCTION BY MASSIVE PARTICLES, Astrophys.J. 352 (1990) 654.

[50] A. C. Vincent and P. Scott, Thermal conduction by dark matter with velocity and momentum-dependent cross-sections, arXiv:1311.2074. 